\title{
Mixing in Magnetized Interiors of Solar-Type Stars: Frequently Asked Questions
}

\author{
Paul Charbonneau \\ HAO/NCAR, P.O. Box 3000, Boulder, CO 80307, U.S.A.
}

\begin{abstract}
This short paper discusses various issues pertaining to the redistribution of angular momentum and mixing of chemical species in the radiative interior of solar-type stars. Results obtained to date indicate that models combining magnetically-mediated angular momentum transport with turbulent mixing of chemical species offer the best hope of explaining the observed rotation and light element evolution in open clusters, without doing excess violence to seismic measurements of the solar internal differential rotation.
\end{abstract}

\section{Introduction}

Models relying on turbulence generated by shear flow instabilities for mixing angular momentum and chemical elements in the radiative interiors of solar-type stars have been reasonably successful at describing parametrically the Lithium abundances and surface rotational evolution observed in young clusters. However, such models suffer from two important failings: once calibrated to reproduce the solar Lithium abundance and present-day surface rotation rate, they (1) leave too strong an angular velocity gradient in the solar radiative interior, in disagreement with helioseismic inferences, and (2) deplete too much Beryllium (e.g., Balachandran, this volume). In a series of papers building on earlier work (e.g., Mestel \& Weiss 1987), my collaborators and I have presented numerical simulations showing that models relying instead on magnetic stresses to internally redistribute angular momentum can overcome these difficulties (Charbonneau \& MacGregor 1992, hereafter CM92; Barnes, Charbonneau \& MacGregor, 1998, hereafter BCM98; MacGregor \& Charbonneau 1999).

Rather than attempting to summarize the content of the aforecited papers, I adopt here a "question-and-answer" format to emphasize the features of magnetized models that bear strongest on the issue of elemental mixing. Unless otherwise noted, questions are posed and answered in the context of rotational evolution models where internal angular momentum redistribution is dominated by magnetic stresses generated by the shearing of a pre-existing large-scale poloidal field pervading the radiative interior, and presumably of fossil origin (see CM92). In such models, the diffusion coefficients needed to compute the mixing of chemical elements are calculated a posteriori using the time-varying differential rotation profiles coming out of the spin-down simulation, using various prescriptions in a manner analogous to what is done by other authors focusing on purely hydrodynamical mixing (see BCM98). 


\section{Why is the present-day solar surface rotation rate largely insen- sitive to details of internal angular momentum redistribution?}

This is due to a general property of angular momentum from thermally-driven, rotating, magnetized winds, for which the rate of angular momentum loss increases rapidly with increasing rotation rate. Consequently, faster rotators spin down faster, and "memory" of the rotational initial condition is lost within $10^{9} \mathrm{yr}$ or so (see, e.g., Keppens, MacGregor \& Charbonneau 1995).

\section{Why is the Sun's core rotation so sensitive to the degree of mag- netic coupling at the core-envelope interface?}

Regions of the radiative interior threaded by poloidal magnetic fieldlines that close upon themselves without crossing the core-envelope interface have a tendency to "decouple" from the envelope rotation, since they can only exchange angular momentum viscously across poloidal flux surfaces. Consequently such regions tend to retain their initial high angular momentum, leading to sustained differential rotation throughout spin-down, in some cases persisting to the solar age. See the video segment accompanying the CM92 paper.

\section{Why is the Sun's core rotation so insensitive to the assumed in- ternal magnetic field strength?}

For a given poloidal field strength and geometry, the system simply builds up whatever toroidal magnetic field it needs to equilibrate the solar wind torque; this occurs on a timescale of the order of the Alfvén time, which even for fields as weak as $1 \mathrm{G}$ is much shorter than any other relevant timescale in the problem. This equilibration is a general feature of spin-down models, and takes place under a wide variety of modelling assumptions. For more on this see $\S 3.5$ of CM92, MacGregor \& Brenner (1991), and MacGregor \& Charbonneau (1994).

\section{Why is the latitudinal differential rotation of the solar convective envelope not imprinted on the radiative core?}

The assumption that it should is a misapplication of Ferraro's isorotation theorem, stating that in an axisymmetrically rotating, inviscid and perfectly conducting magnetized radiative interior, the only allowed steady-states must satisfy $\mathbf{B}_{p} \cdot \nabla \Omega=0$, i.e., the angular velocity $\Omega$ is constant along a line of force of the poloidal magnetic field $\mathbf{B}_{p}$. The situation in the solar radiative interior is far more complex, however. The buildup of a radial shear at the core-envelope interface leads to the formation of a magnetoviscous boundary layer where dissipative processes do play an important role, even if the viscosity and magnetic diffusivity are both very small. Whether and how much of the envelope differential rotation ends up imprinted on the radiative core depends crucially on the degree of magnetic coupling at the core-envelope interface. For more on this and related matters, see Rüdiger \& Kitchatinov (1997) and MacGregor \& Charbonneau (1999). 


\section{Why are the present-day solar light element abundances so sen- sitive to the assumed internal magnetic field geometry?}

As spin-down proceeds, regions of the radiative interior that are threaded by fieldlines that cross the core-envelope interface rapidly reach a state of nearrigid rotation. The absence of significant angular velocity gradients, in turn, leads to very low diffusion coefficients, and so to weak turbulent transport of chemical species. Such regions thus act as reservoirs of light elements, which can be tapped into and in some cases lead to increases in the surface $\mathrm{Li}$ and Be abundances at later evolutionary epochs. This is a $2 \mathrm{D}$ effect that has no counterpart in 1D models. See BCM98, $§ 3.2$ and Figs. 4, 5.

\section{Why are the present-day solar light element abundances so sen- sitive to the assumed internal magnetic field strength?}

Recall that the shear at the core-envelope interface will build up until a strong enough toroidal field has been induced so that the associated Lorentz force equilibrates the applied torque. For weaker poloidal fields, a stronger toroidal component must be produced, which requires more time, so that a stronger rotational shear has time to build up at the core-envelope interface. Everything else being equal, this stronger shear leads to enhanced turbulent transport, and stronger depletion of light elements. See $\S 3.2$ and Fig. 6 in BCM98.

\section{Why do magnetic models deplete less Be (relative to $\mathrm{Li}$ )?}

In models relying on magnetic stresses to redistribute angular momentum, strong angular velocity gradients are maintained only near the core-envelope interface, in contrast to hydrodynamical models, where significant $\Omega$-gradients persist all the way down to the deep core. Consequently, the efficiency of turbulent mixing decreases more rapidly with depth in magnetized models than it does in the purely hydrodynamical case, so that once the model is adjusted to reproduce Lithium, less Beryllium is depleted. See Fig. 8 of BCM98, and accompanying discussion (but also Brun, and Zahn, this volume).

\section{References}

Barnes, G., Charbonneau, P., \& MacGregor, K.B. 1992, ApJ, (BCM98)

Charbonneau, P., \& MacGregor, K.B. 1992, ApJ, (CM92)

Keppens, R., MacGregor, K.B., \& Charbonneau, P. 1995, A\&A, 294, 469

MacGregor, K.B., \& Brenner, M. 1991, ApJ, 376, 204

MacGregor, K.B., \& Charbonneau, P. 1994, in ASP Conf. Ser. Vol. 64, Cool Stars, Stellar Systems, and the Sun, ed. J.-P. Caillault (San Francisco: ASP), 174

MacGregor, K.B., \& Charbonneau, P. 1999, ApJ, 519, 911

Mestel, L., \& Weiss, N.O. 1987, MNRAS, 226, 123

Rüdiger, G., \& Kitchatinov, L.L. 1997, Astron. Nachr., 318, 273 